\title{
ERRATUM
}

\section{Gram-positive Rhizobacterium Bacillus amyloliquefaciens FZB42 Colonizes Three Types of Plants in Different Patterns}

\author{
Ben Fan $^{1,2 *}$, Rainer Borriss ${ }^{2}$, Wilfrid Bleiss ${ }^{3}$, and Xiaoqin $\mathrm{Wu}^{1}$ \\ ${ }^{1}$ Institute of Forest Protection, Nanjing Forestry University, Nanjing 210037, P. R. China \\ ${ }^{2}$ Institut fur Biologie/Bakteriengenetik, Humboldt Universitat Berlin, Chausseestrasse 117, D-10115 Berlin, Germany \\ ${ }^{3}$ Institut fur Biologie/Molekulare Parasitologie, Humboldt-Universitat Berlin, Philippstrasse 13, D-10115 Berlin, Germany
}

In the article by Fan et al. that appears in the Journal of Microbiology 2012; 50, 38-44. The following Fig. 3 was inadvertently missing.
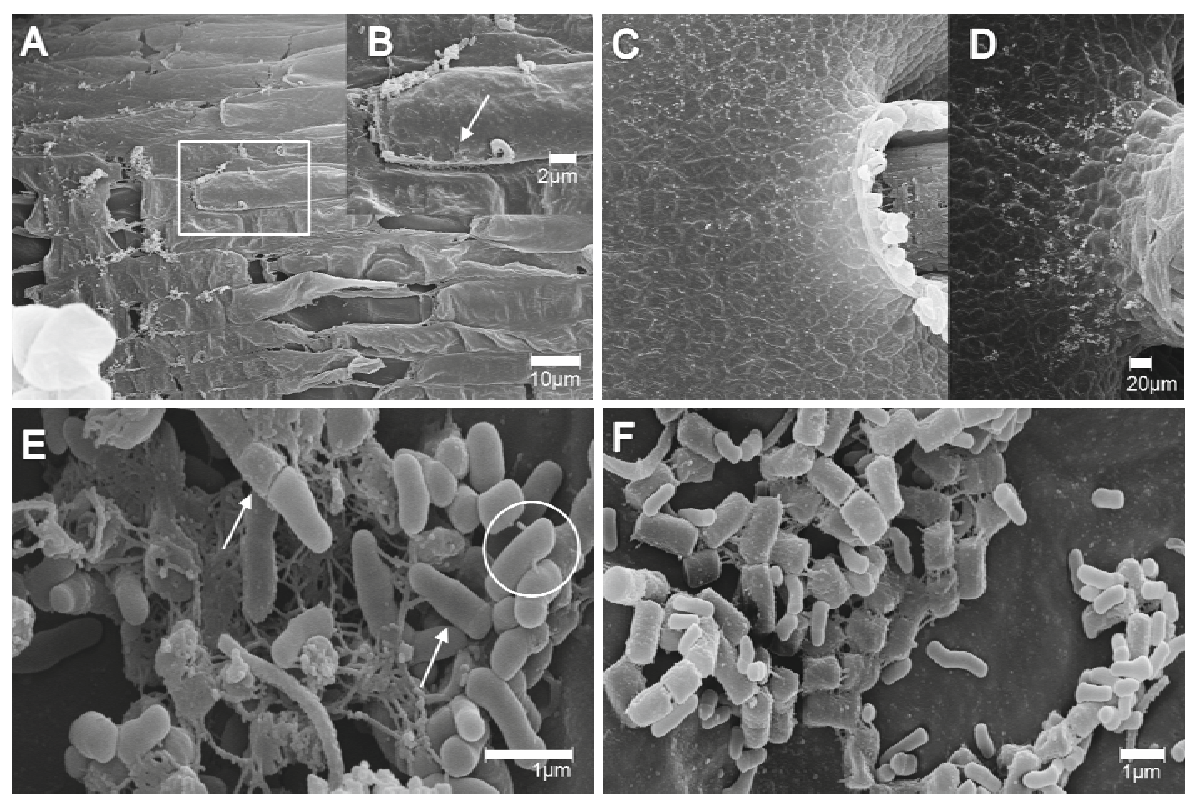

Fig. 3. SEM micrographs of B. amyloliquefaciens FZB42 colonizing L. minor 9 days after inoculation in Steinberg medium. Panel A\&B showed the colonization of FZB42 on Lemna roots. Panel B is an amplified view of the area enclosed by the rectangle in (A). Note the bacterial cells populating along the grooves between the epidermal cells and the presumed root exudates indicated by the arrow in (B). (C\&D) showed the colonization of FZB42 on the ventral surface of Lemna fronds near the frond-root linking region. Note that most FZB42 cells populated along the sunken spaces between parenchyma cells. (E\&F) showed some details of the biofilms formed by FZB42 in the ventral side of Lemna fronds. Note the altering shape of FZB42 cells indicated by the arrows and the fiber structures linking the bacteria together in (E) and the altered shape of many FZB42 cells in (F) and their rough coating structures. 